Few-Body Systems manuscript No.

(will be inserted by the editor)

F.F. Van der Veken · N.G. Stefanis

\title{
Identifying the Universal Part of TMDs
}

Received: date / Accepted: date

\begin{abstract}
We attempt to identify a path layout in the definition of transverse-momentum-dependent T-odd parton distribution functions (TMD)s which combines features of both, initial- and final-state interactions, so that it remains universal despite the fact that the Wilson lines entering such TMDs change their orientation. The generic structure of the quark correlator for this path layout is calculated.
\end{abstract}

Keywords Wilson lines · SIDIS and DY TMDs · Universal path layout

\section{Introduction}

Collinear factorization is a fully grown and well understood framework to describe inclusive processes like deep inelastic scattering (DIS). The hadron is portrayed by a parton distribution function (PDF) which only depends on the longitudinal momentum fraction $x$ shared by the struck quark, as the transversal momentum components are integrated out. Factorization is then achieved by convoluting the PDF with the hard part of the interaction and integrating over $x$, see [1] for a recent review.

The collinear framework is however less suitable to describe high-energy particle collisions that are not fully inclusive. This is, because in this case one has to take into account the transversal part of the quark momentum as well. This enlarged framework is based on $\boldsymbol{k}_{\perp}$-factorization and employs PDFs which depend on the transverse momentum - thus transverse-momentum-dependent PDFs or TMDs for short (see 2] for an exhaustive review). Exclusive processes are extremely hard to treat because one deals with the full nonperturbative quark-gluon dynamics which cannot be computed from first principles and has to be modeled. However, for some processes which are semi-inclusive and the set of the final states is not fully integrated out but is neither fully identified, factorization theorems have been proven. Among them, the most well known example is semi-inclusive deep inelastic scattering (SIDIS), which has been thoroughly treated in [3; 4 ; ; 5 . However, a few years later it was realized due to the non-vanishing Sivers effect 6] that the presence of the Wilson line inside the TMD definition should be taken into consideration [7]. The reason is that the Wilson line entails the existence of T-odd TMDs, breaking the universality of the TMD. Indeed, while in SIDIS the Wilson line is future-pointing, in initial-state processes like the Drell-Yan (DY), where a produced virtual photon decays into a muon pair, the Wilson line is past-pointing, leading to an overall sign change for T-odd TMDs [8; 9; 10]. This

Presented by the first author at Light-Cone 2015, September 21-25, 2015, Frascati, Italy.

F.F. Van der Veken

Departement Fysica, Universiteit Antwerpen, B-2020 Antwerpen, Belgium

E-mail: frederik.van.der.veken@cern.ch

Present address: CERN, (Meyrin site), Building 9, Room 1-012, CH-1211 Geneve 23, Switzerland

N.G. Stefanis

Institut für Theoretische Physik II, Ruhr-Universität Bochum, D-44780 Bochum, Germany

E-mail: stefanis@tp2.ruhr-uni-bochum.de 


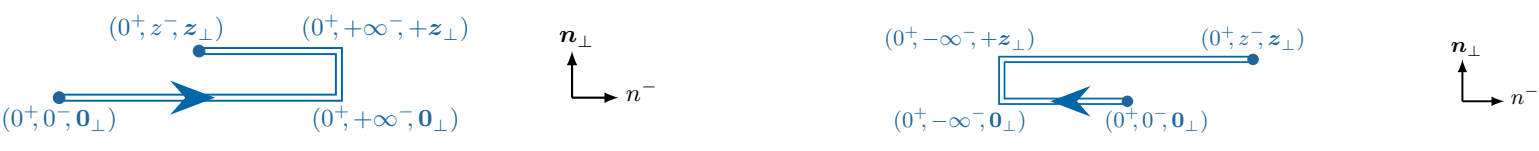

Fig. 1 Path layouts pertaining to SIDIS (left) and the Drell-Yan process (right)

came as a surprise because the universality of the TMD was presumed taking recourse to the stringent validity of collinear factorization in DIS.

This important finding notwithstanding, it appears attractive to ask whether it is possible to create a path layout that combines features of both initial and final state interactions. Such a geometrical structure of Wilson lines could eventually be associated with a common and hence universal part of the SIDIS and the DY processes. This paper is devoted to the conceptual investigation of this issue. The treatment of the renormalization issues of the correlators, in particular questions related to the geometrical features of the Wilson lines - the appearance of cusps and their anomalous dimensions - is relegated to the literature (see, for instance, 2 ; $11 ;$; $12 ; 13]$ and references cited therein). A more general assessment and a fuller treatment of the present approach will be given elsewhere. The work is organized as follows. In the next section, we recall the structure of the gauge-invariant quark correlator. The new path layout is discussed in Sec. 3. whereas the generic quark correlator employing the new path layout is considered in Sec. 4. Finally, Sec. 5] contains our main conclusions.

\section{Gauge-invariant quark correlator}

The key quantities to describe the three-dimensional dynamics of partons within hadrons, e.g., a proton, in high-energy collisions is provided by TMD distribution and fragmentation functions. Typically, these quantities depend on a large scale, say, $Q^{2}$, which can be the invariant mass in a Drell-Yan process, and $q_{\perp}^{2} \ll Q^{2}$, the transverse momentum of the lepton pair in the same process (with analogous definitions for SIDIS). The generic form of the TMD quark correlator is given by

$$
\Phi_{i j}^{q[C]}\left(x, \mathbf{k}_{\perp} ; n\right) \sim \int \frac{d z^{-} d^{2} \mathbf{z}_{\perp}}{(2 \pi)^{3}} e^{-i k^{+} z^{-}+i \boldsymbol{k}_{\perp} \cdot \boldsymbol{z}_{\perp}}\left\langle p\left|\bar{\psi}_{j}(z) \mathcal{U}(0 \rightarrow z \mid C) \psi_{i}(0)\right| p\right\rangle_{z^{+}=0}
$$

where the Wilson line to ensure gauge invariance is a path-ordered exponential of the form

$$
\mathcal{U}(a \rightarrow b \mid C)=\mathcal{P} \exp \left[i g \int_{a}^{b} d z \cdot A(z)\right]
$$

and the path $C$ has to be taken along the color flow from $a$ to $b$ for each considered process.

As already mentioned, for processes with final-state interactions (SIDIS) the path wraps around at $+\infty$ (left panel of Fig. 1), whereas for processes with initial-state interactions (DY process) — right panel of Fig. 1 - the path wraps around $-\infty$. It can be shown [14; 15; 16] that changing the endpoint of a linear Wilson-line segment from $+\infty$ to $-\infty$ (or vice versa) amounts to the substitution $n \rightarrow-n$ (where $n$ is the directional vector of the segment), i.e., $\mathcal{U}(a \rightarrow+\infty \mid n)=\mathcal{U}(a \rightarrow-\infty \mid-n)$. Because the Wilson line is the only factor with an $n$-dependence, this relation is repeated in the correlator:

$$
\left.\Phi_{i j}^{q[C]}\left(x, \mathbf{k}_{\perp} ; n\right)\right|_{\mathrm{DY}}=\left.\Phi_{i j}^{q[C]}\left(x, \mathbf{k}_{\perp} ;-n\right)\right|_{\mathrm{SIDIS}} .
$$

The fully unintegrated quark correlator can be parameterized by a set of scalar functions that are, after the $k^{-}$-integration, related to their TMD counterparts 17]. The Lorentz structure of the correlator will only depend on contractions of the vectors $p^{\mu}, k^{\mu}, S^{\mu}$ and $n^{\mu}$ with themselves and with the Dirac basis. In the case of an unpolarized proton the fully unintegrated correlator can be cast in the form

$$
\begin{aligned}
\Phi(p, k, n)=M A_{1} & +\gamma_{\mu}\left(p^{\mu} A_{2}+k^{\mu} A_{3}+\frac{M^{2}}{p \cdot n} n^{\mu} B_{1}\right)+\gamma_{\mu} \gamma_{5} \frac{1}{p \cdot n} \varepsilon^{\mu \nu \rho \sigma} n_{\nu} p_{\rho} k_{\sigma} B_{4} \\
& +i \gamma_{\mu \nu}\left[\frac{1}{M} p^{\mu} k^{\nu} A_{4}+\frac{M}{p \cdot n}\left(p^{\mu} B_{2}+k^{\mu} B_{3}\right) n^{\nu}\right] .
\end{aligned}
$$




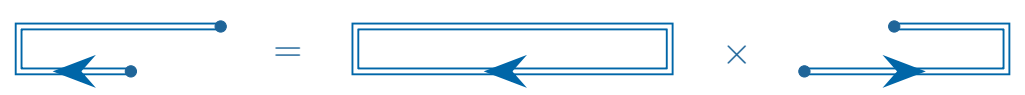

Fig. 2 Relation between the DY path layout (left-hand side) and that in SIDIS (right-hand side)

The important thing to notice here is that the directional vector $n_{\mu}$ appears scaleless, i.e., only via factors of the form $\frac{n^{\mu}}{p \cdot n}$, being straightforward to show that this remains true in the polarized case as well. As the $k^{-}$-integration does not influence $n^{\mu}$, it is tempting to conclude that the TMD correlator is invariant under a sign change in $n$, and hence universal by virtue of Eq. (3). However, one should not forget that the scalar functions themselves can still depend on $n^{\mu}$. Moreover, it has been demonstrated that those functions that are odd under time reversal flip sign when interchanging past-pointing with future pointing Wilson lines [7]. This can be summarized in terms of the following relations

$$
f_{\mathrm{T} \text {-even }}^{\mathrm{DY}}=f_{\mathrm{T} \text {-even }}^{\mathrm{SIDIS}}, \quad f_{\mathrm{T} \text {-odd }}^{\mathrm{DY}}=-f_{\mathrm{T} \text {-odd }}^{\mathrm{SIDIS}} .
$$

\section{New path layout}

In what follows we will try to construct a path layout that encapsulates features of both initial- and final-state interactions in a single frame. First we note that due to the transitivity property of a Wilson line, the layouts of SIDIS and DY can be easily related to one another (see Fig. 2):

$$
\mathcal{U}^{\mathrm{DY}}=\mathcal{U}^{\text {loop }} \mathcal{U}^{\text {SIDIS }}
$$

where $\mathcal{U}^{\text {loop }}$ is a loop with infinite longitudinal length (in the light-cone minus direction), and with finite transversal width (equal to $\mathbf{z}_{\perp}$ ). The overlapping parts cancel out on the right-hand side of Eq. (6), resulting in the DY path layout. However, this relation is not reproduced at the level of the correlator $\Phi^{\mathrm{DY}} \neq \Phi^{\text {loop }} \Phi^{\text {SIDIS }}$ because of the intricacies related to the splitting of expectation values, so that in general

$$
\left\langle\mathcal{U}^{\text {loop }} \mathcal{U}^{\text {SIDIS }}\right\rangle \neq\left\langle\mathcal{U}^{\text {loop }}\right\rangle\left\langle\mathcal{U}^{\text {sIDIS }}\right\rangle
$$

We could at most hope that this relation holds up to power corrections in $\alpha_{s}$ and/or $N_{c}$. Instead, we split the loop contour at transverse infinity into parts, each in turn assigned to one of the two considered path layouts so that one obtains the following configuration:

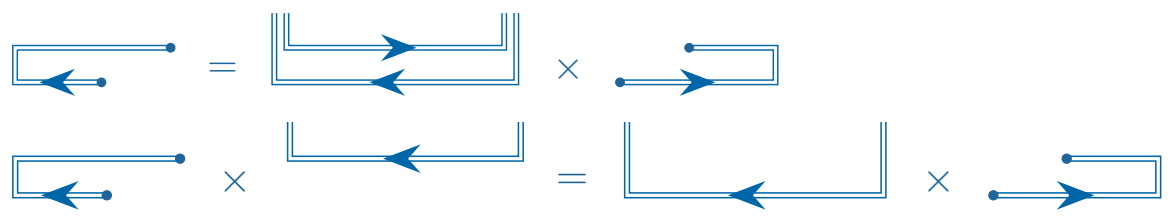

The emerging layout represents a new contour pattern which can be conceived of as being the "golden mean" between the SIDIS and the DY path layouts. Its structure is illustrated in Fig. 3 segments 1 and 2 correspond to the part on the left of the final-state cut in the DY layout, while segments 4 and 5 refer to the part on the right of the cut in the SIDIS layout. Segment 3 vanishes in Lorentz and axial gauges, but is needed for gauge invariance and in order to ensure that the full layout reduces to the regular PDF layout after performing the $\mathbf{k}_{\perp}$-integration (a simple straight line). Let us emphasize that we do not pretend that the correlator built from this layout should be considered as a physical quantity. It is merely a mathematical construct out of academic interest, trying to represent the core universal part of the regular quark correlator. If we need to introduce a final-state cut, we draw it somewhere through segment 3 , the exact splitting point being irrelevant for practical calculations.

\section{Generic quark correlator for the new path layout}

The next step is to retrieve the quark correlators for SIDIS and DY from our new correlator. We denote the latter, i.e., the correlator constructed from our new path layout, as $\tilde{\Phi}$, so that we can write the original correlators as a convolution between a (process-dependent) correction factor and $\tilde{\Phi}$ :

$$
\Phi^{\mathrm{SIDIS}}=C^{\mathrm{SIDIS}} \otimes \tilde{\Phi}, \quad \Phi^{\mathrm{DY}}=C^{\mathrm{DY}} \otimes \tilde{\Phi},
$$




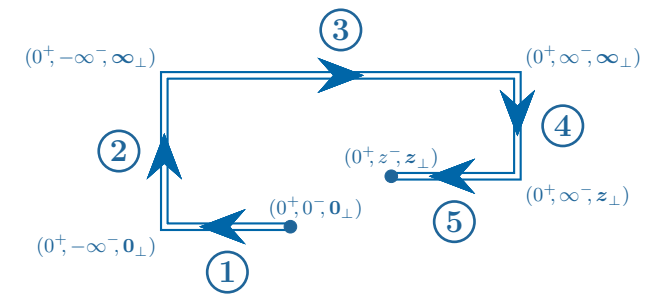

Fig. 3 The proposed new path layout for the Wilson line

where the $C^{i}$ are expected to be perturbative quantities. In leading order, all Wilson lines reduce to unity and hence $\Phi^{\mathrm{SIDIS}}=\Phi^{\mathrm{DY}}=\tilde{\Phi}$. This implies that the correction factors at the leading-order level are just delta functions:

$$
C_{0}^{\text {SIDIS }}=C_{0}^{\text {DY }}=\delta(1-x) \delta^{(2)}\left(\mathbf{k}_{\perp}\right) .
$$

A fruitful approach to continue to higher orders, is to focus on parameters which are peculiar to each of the three correlators, $\Phi^{\mathrm{SIDIS}}, \Phi^{\mathrm{DY}}, \tilde{\Phi}$. In Feynman gauge (or another Lorentz, i.e., covariant gauge), only segments 1 and 5 in Fig. 3 survive. What changes across different correlators is the orientation of these segments, as depicted graphically in Table 1. To maximize reproducibility, we assign different directional vectors $n_{1}$ and $n_{5}$ to these segments. This allows the retrieval of each correlator by a mere substitution of $n_{1,5}$. Similarly, in the light-cone gauge (or any other axial gauge), only segments 2 and 4 contribute, and the differing parameter is the transversal endpoint $r^{-}$. Hence, we assign different endpoints $r_{2}$ and $r_{4}$ to these segments.

In what follows, we perform our investigation of the parametric structure in Feynman gauge; the extension to the light-cone gauge is, however, trivial. Expanding Eqs. (10) in terms of powers of $\alpha_{s}$, it is easy to show that the first-order correction factors depend on the relevant substitution differences:

$$
\left.C_{1}^{\text {SIDIS }} \sim \Phi_{1}\right|_{n_{1} \rightarrow-n_{1}}-\Phi_{1},\left.\quad \quad C_{1}^{\mathrm{DY}} \sim \Phi_{1}\right|_{n_{5} \rightarrow-n_{5}}-\Phi_{1},
$$

where $\Phi$ is the generic correlator, i.e., with both $n_{1}$ and $n_{5}$ left unspecified. Now to continue, we note that the generic correlator in leading order of $\alpha_{s}$ consists of three parts: a term not depending on the directional vector, and terms "even" or "odd" in $n$ 1

$$
\Phi=\Phi_{0}+\alpha_{s}\left(A+\frac{n_{1}+n_{5}}{2} B+\frac{n_{1}-n_{5}}{2} C\right)+\mathcal{O}\left(\alpha_{s}^{2}\right)
$$

where $A, B$, and $C$ are the same for all three correlators. Comparing this generic form with Table 1 . we observe that

$$
\begin{aligned}
\tilde{\Phi} & =\Phi_{0}+\alpha_{s}\left(A-n^{-} B\right)+\mathcal{O}\left(\alpha_{s}^{2}\right), & C_{1}^{\mathrm{SIDIS}} & =-\alpha_{s} n^{-}(B+C), \\
\Phi^{\mathrm{SIDIS}, \mathrm{DY}} & =\Phi_{0}+\alpha_{s}\left(A \pm n^{-} C\right)+\mathcal{O}\left(\alpha_{s}^{2}\right), & C_{1}^{\mathrm{DY}} & =-\alpha_{s} n^{-}(B-C) .
\end{aligned}
$$

It is important to realize that $A$ is the only term that is T-even, with both $B$ and $C$ being T-odd. If $B$ would vanish, then $\tilde{\Phi}$ would be completely universal. A deep investigation of this term would help to shed light on the universality properties of $\tilde{\Phi}$ and TMDs in more general terms.

\section{Conclusions}

We have constructed a new path layout, which combines features of both initial- and final-state interactions entering the calculation of quark correlators in SIDIS and DY-type processes making use of Wilson lines to preserve gauge invariance. The ensuing generic correlator provides a theoretical tool for the investigation of the universality properties of the original correlators considered in the SIDIS and Drell-Yan processes in connection with particular process-dependent contours [17; 18]. The proposed path layout reveals universal features and enhances the insight into the universality properties of the

\footnotetext{
1 No other terms appear at the first-order level due to $\left(n_{1}\right)^{2}=\left(n_{5}\right)^{2}=n_{1} \cdot n_{5}=0$. One can keep the "even/odd" distinction at higher orders using the sign of $n_{1}$ and $n_{5}$ instead of employing the vectors themselves.
} 


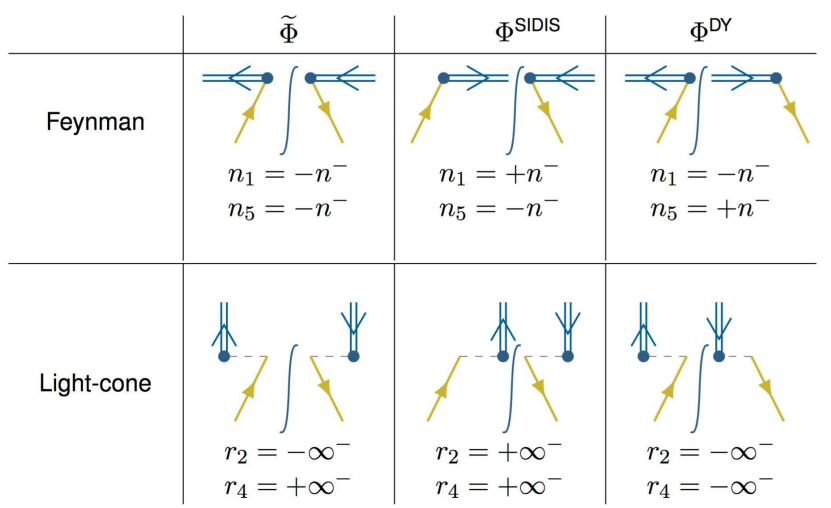

Table 1 Values of the parameters $n_{1}, r_{2}, r_{4}$ and $n_{5}$ entering the various considered correlators and pertaining to two different gauges. The double lines denote Wilson lines

known correlators. Adopting an approach which employs two parameters, we were able to perform explicit calculations of these correlators in the Feynman and also in the light-cone gauge and demonstrate the usefulness of the proposed layout. A more general investigation of the universal features of TMDs, which includes the renormalization properties of the correlators, will be given elsewhere.

Acknowledgements F. F. Van der Veken gratefully acknowledges support from the Gary T. McCartor Fund Fellowship and thanks the Research Foundation-Flanders (FWO) for a travel grant. He is also thankful to the members of the Institute for Theoretical Physics II of Bochum University for their warm hospitality during a research stay when most part of this investigation was carried out.

\section{References}

1. Brambilla, N., et al.: QCD and Strongly Coupled Gauge Theories: Challenges and Perspectives. Eur. Phys. J. C 74, $2981(2014)$

2. Boer, D., et al.: Gluons and the quark sea at high energies: Distributions, polarization, tomography. arXiv:1108.1713 [nucl-th].

3. Mulders, P.J., Tangerman, R.D.: The complete tree level result up to order 1/Q for polarized deep inelastic leptoproduction. Nucl. Phys. B 461, 197 (1996); Erratum: ibid. B 484, 538 (1997)

4. Boer, D., Mulders, P.J.: Time reversal odd distribution functions in leptoproduction. Phys. Rev. D 57, 5780 (1998)

5. Boer, D., Jakob, R., Mulders, P.J.: Angular dependences in electroweak semiinclusive leptoproduction. Nucl. Phys. B 564, $471(2000)$

6. Brodsky, S.J., Hwang, D.S., Schmidt, I.: Final state interactions and single spin asymmetries in semiinclusive deep inelastic scattering. Phys. Lett. B 530, 99 (2002)

7. Collins, J.C.: Leading twist single transverse-spin asymmetries: Drell-Yan and deep inelastic scattering. Phys. Lett. B 536, 43 (2002)

8. Ji, X.-d., Yuan, F.: Parton distributions in light cone gauge: Where are the final state interactions?. Phys. Lett. B 543, 66 (2002)

9. Belitsky, A.V., Ji, X., Yuan, F.: Final state interactions and gauge invariant parton distributions. Nucl. Phys. 656, 165 (2003)

10. Boer, D., Mulders, P.J., Pijlman, F.: Universality of T odd effects in single spin and azimuthal asymmetries. Nucl. Phys. B 667, 201 (2003)

11. Collins, J.C.: Foundations of perturbative QCD. Cambridge University Press, Cambridge (2011)

12. Cherednikov, I.O., Stefanis, N.G.: Wilson lines and transverse-momentum dependent parton distribution functions. A Renormalization-group analysis. Nucl. Phys. 802, 146 (2008)

13. Stefanis, N..G.: Worldline techniques and QCD observables. Acta Phys. Polon. Supp. 6, 71 (2013)

14. Van der Veken, F.F.: Working With Wilson Lines. Int. J. Mod. Phys. Conf. Ser. (2015), in print

15. Van der Veken, F.F.: A new approach to piecewise linear Wilson lines. Int. J. Mod. Phys. Conf. Ser. 37 (2015) 1560029

16. Van der Veken, F.F.: Piecewise Linear Wilson lines. EP.J Web Conf. 85 (2015) 02004

17. Goeke, K., Metz, A., Schlegel, M.: Parameterization of the quark-quark correlator of a spin- $1 / 2$ hadron. Phys. Lett. B 618 (2005) 90

18. Bacchetta, A., Mulders, P.J., Pijlman, F.: New observables in longitudinal single-spin asymmetries in semi-inclusive DIS. Phys. Lett. B 595 (2004) 309 